\title{
Primeros contactos con los materiales de construcción de los estudiantes de arquitectura.
}

\section{First contacts with the building materials of the architecture students.}

EDUARDO DIZ-MELLADO

ORCID: https://orcid.org/0000-0002-2039-1307

email:ediz@us.es

Universidad de Sevilla.

Escuela Técnica Superior de Arquitectura.

Departamento de Construcciones

Arquitectónicas I

DOI: http://dx.doi.org/10.12795/9788447231003.027

Pp.: 573-592 


\section{Contexto}

El curso de innovación docente se plantea sobre la asignatura de Construcción $\mathbf{1}$, perteneciente al primer curso del Grado en Fundamentos de Arquitectura. El objetivo fundamental de la asignatura de Construcción 1 es transmitir los primeros conocimientos sobre la construcción en la arquitectura, así como reconocer diferentes familias de materiales. Se trata, a priori, de la primera toma de contacto de los alumnos con la construcción.

La asignatura se organiza en módulos de dos horas, con dos clases a la semana. Se trata normalmente de clases teórico-prácticas en su mayoría, en las que dos horas suelen dedicarse exclusivamente a teoría, y las otras dos a trabajo práctico en el aula de forma tutelada. Paralelamente, en tres sesiones, los alumnos tienen docencia práctica en el laboratorio de construcción, con seis horas presenciales durante el cuatrimestre, en las que se desarrollará el ciclo mejora en el aula (CIMA). Las prácticas de laboratorio a su vez tienen un contenido teórico principal, y una parte práctica para el desarrollo de los alumnos. El desarrollo teórico está basado en un contenido que engloba diferentes familias de materiales y sistemas constructivos, y el contenido práctico consiste en la realización de probetas con algunos materiales explicados en la teoría. Durante el ejercicio práctico, los alumnos se enfrentan por primera vez a la realización de probetas de pasta de yeso, mortero de cal o cemento. Tras la realización de las probetas se procede a la rotura de las mismas iniciando un debate común sobre los resultados. De esta forma se fomenta el desarrollo de un pensamiento crítico. Se trata por tanto de aplicar los contenidos teóricos explicados en clase a la práctica.

Las clases se desarrollan en grupos de 27 alumnos, divididos en nueve subgrupos por clase de tres personas para el desarrollo de la práctica. La docencia se imparte a todos los grupos de primero del Grado en Fundamentos

Ciclos de Mejora en el Aula (2020). Experiencias de Innovación Docente de la US Esta obra se distribuye con la licencia Creative Commons 
de Arquitectura en horario de mañana y tarde durante el primer cuatrimestre, en módulos de dos horas. La organización de la clase trata hasta el momento de una hora y media de contenido teórico y media hora de elaboración de la práctica. Es por esto, que el objetivo principal del diseño y aplicación del Ciclo de Mejora sea convertir las prácticas de laboratorio en clases realmente prácticas. El CIMA se plantea para tres sesiones de dos horas en las que se abordarán contenidos como conglomerantes y conglomerados, sistemas constructivos y productos.

\section{Diseño del CIMA}

El diseño del CIMA se realiza teniendo en cuenta los principios didácticos referidos a la clasificación previa y adecuación de los contenidos, la reflexión del modelo metodológico habitual y propuesta mediante una nueva metodología, así como con la evaluación y comparación inicial y final del aprendizaje conseguido por los alumnos.

\section{Contenidos}

Los contenidos de la asignatura se han organizado y jerarquizado previo al diseño del mapa conceptual de las sesiones (Tabla 1). Posteriormente, se detalla en la Figura 1 el mapa de contenidos diseñado para la aplicación del CIMA. En el mapa se interrelacionan los contenidos estructurantes y secundarios con el fin de lograr un discurso único durante las sesiones. Se distinguen varios grupos de contenidos (Porlán Ariza, 2017), con contenidos de tipo dato, concepto, procedimientos (psicomotrices), o con una finalidad o valor determinado.

Ciclos de Mejora en el Aula (2020). Experiencias de Innovación Docente de la US Esta obra se distribuye con la licencia Creative Commons 
Tabla 1. Organización y jerarquización de los contenidos.

\begin{tabular}{|c|c|c|c|}
\hline \multirow{4}{*}{ P1 } & $\begin{array}{l}\text { Definición de conglomerantes } \\
\text { y conglomerados }\end{array}$ & Datos / Conceptos & Estructurante \\
\hline & $\begin{array}{l}\text { Proceso de fabricación } \\
\text { conglomerantes }\end{array}$ & Conceptos/Valores & Secundario \\
\hline & Aplicación de conglomerados & $\begin{array}{l}\text { Procedimientos / } \\
\text { Finalidad }\end{array}$ & Secundario \\
\hline & Fabricación de probetas & $\begin{array}{l}\text { Procedimental } \\
\text { Psicomotriz }\end{array}$ & Secundario \\
\hline P2 & $\begin{array}{l}\text { Diseño y montaje de sistemas } \\
\text { constructivos }\end{array}$ & $\begin{array}{l}\text { Datos / Conceptos } \\
\text { / Procedimental } \\
\text { Psicomotriz }\end{array}$ & Estructurante \\
\hline \multirow{6}{*}{ P3 } & Tipos de aislantes térmicos & Datos / Conceptos & Secundario \\
\hline & $\begin{array}{l}\text { Aplicación de aislantes } \\
\text { térmicos }\end{array}$ & $\begin{array}{l}\text { Procedimientos / } \\
\text { Valores }\end{array}$ & Estructurante \\
\hline & Tipos de aislantes acústicos & Datos / Conceptos & Secundario \\
\hline & $\begin{array}{l}\text { Aplicación de aislantes } \\
\text { acústicos }\end{array}$ & $\begin{array}{l}\text { Procedimientos / } \\
\text { Valores }\end{array}$ & Secundario \\
\hline & Láminas de protección & Datos / Conceptos & Secundario \\
\hline & Ensayos. Diferentes resultados. & $\begin{array}{l}\text { Datos / Conceptos } \\
\text { / Procedimental } \\
\text { Psicomotriz / Valores }\end{array}$ & Secundario \\
\hline
\end{tabular}

Ciclos de Mejora en el Aula (2020). Experiencias de Innovación Docente de la US Esta obra se distribuye con la licencia Creative Commons 


\section{EDUARDO DIZ-MELLADO}

\section{¿Cómo se construye?}

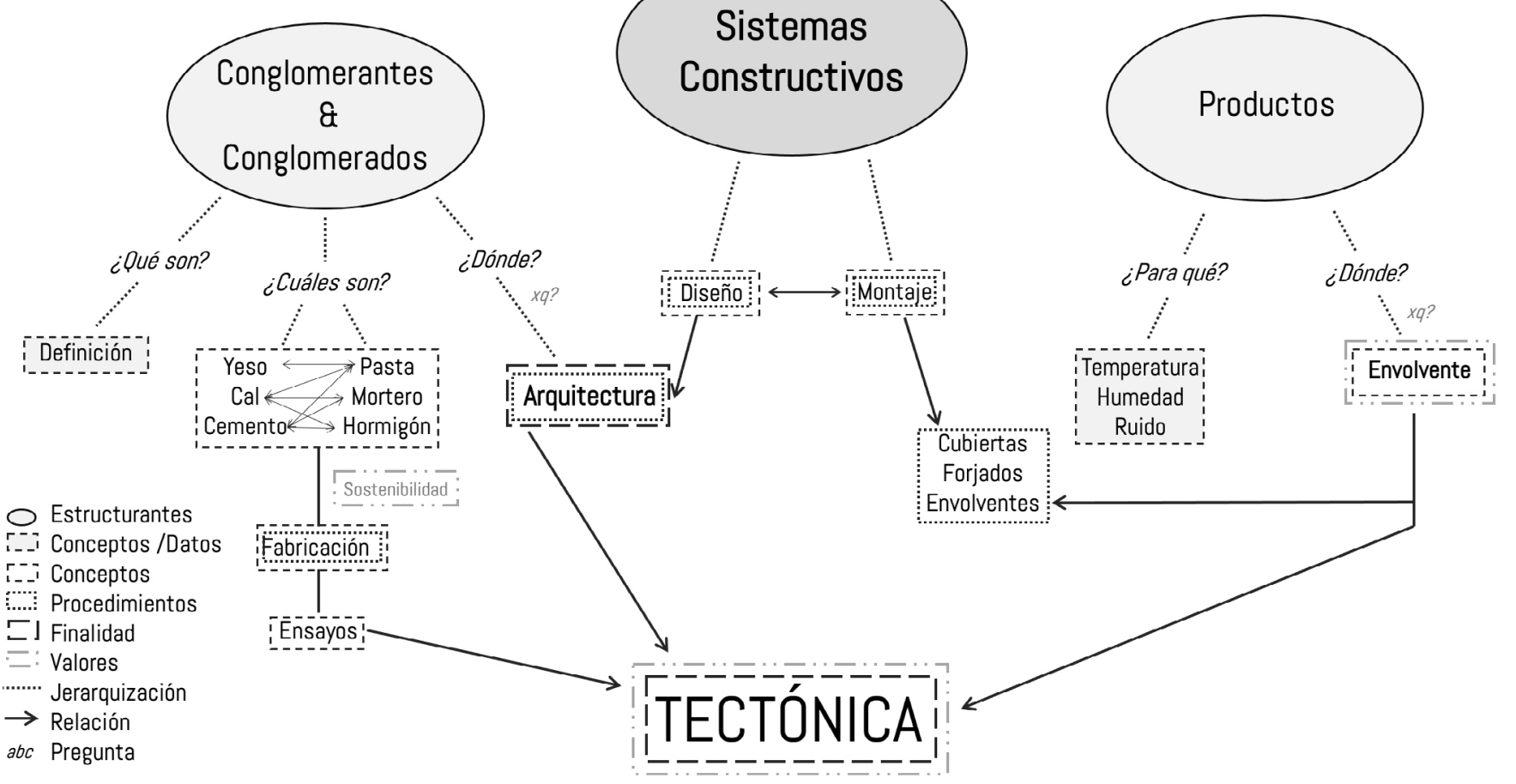

Figura 1. Mapa de contenidos.

Ciclos de Mejora en el Aula (2020). Experiencias de Innovación Docente de la US (c) (i) $\Theta$ Esta obra se distribuye con la licencia Creative Commons 


\section{Diseño del modelo metodológico}

El diseño del modelo metodológico (De Alba y Porlán, 2017) y su justificación, adquieren mayor sentido si primeramente se expone el modelo habitual, con una descripción pormenorizada del mismo. La práctica habitual, consiste en un paquete inicial de diez minutos, en el que los alumnos aprovechan para preguntar dudas de clases o trabajos anteriores, y otros más rezagados llegan a clase. El resto de las dos horas consisten normalmente en una presentación teórica del profesor de aproximadamente una hora y cuarto, en la que intento lanzar preguntas para establecer un diálogo continuo con los alumnos, e incitarles con experiencias de la calle llevadas a clase para que entiendan la problemática real. La segunda parte de la clase, normalmente los últimos 30 minutos, el tiempo se dedica por completo a la elaboración de la práctica de los alumnos por grupo. Se trata de un modelo de docencia establecido previamente.

\section{¿Es realmente una práctica, o se llama así porque se imparte en un laboratorio?}

La docencia considerada práctica de laboratorio debe ser llevadas a la práctica como tal, es decir, el engranaje de unas partes y otras de la clase debe ser fluido. Englobar los contenidos y actividades bajo una misma área será fundamental para afrontar el problema. Introducir un mayor rango temporal a ideas de los alumnos, en cuanto a aplicación de los contenidos aprendidos en situaciones interesantes para ellos, debe tenerse en cuenta (Finkel, 2008). Este tipo de actividades es posible que les ayude a focalizar un tema importante como la vinculación directa entre los contenidos teóricos estudiados en la asignatura y su aplicación en la construcción real.

Paralelamente, la asignatura en la que se aplica tiene la particularidad como se ha descrito previamente, que los

Ciclos de Mejora en el Aula (2020). Experiencias de Innovación Docente de la US Esta obra se distribuye con la licencia Creative Commons 
alumnos realizan puntualmente tres visitas al laboratorio durante el cuatrimestre, lugar donde se aplicará el ciclo de mejora. Se pretende, como se refleja en el esquema de la Figura 2, que la propia docencia del laboratorio en su conjunto sea una actividad de contraste global que acentúe la rama más práctica y constructiva real de la asignatura. La metodología, aunque se describe con más detalle en la secuencia detallada y temporalizada de las actividades diseñadas, pretende una parte introductoria en el que se pregunten dudas sobre materiales vistos en clase, u otros aspectos relevantes que puedan surgir en las visitas al laboratorio. Siempre se les explica que el laboratorio pueden utilizarlo durante los seis años de carrera como una biblioteca de materiales, los cuales pueden ver y tocar cada vez que necesiten, por lo que previo a la primera visita, se les incita a reflexionar e investigar sobre materiales que quieran conocer en profundidad durante la práctica. Posteriormente se les explica el modelo de aprendizaje que se va a impartir en el aula. El contenido teórico se llevará a cabo mediante la visualización de vídeos cortos que se han elaborado previamente como material complementario. Dichos vídeos resumen el contenido teórico de otros años en pocos minutos de forma clara, concisa y atractiva para los alumnos. Comentarios y puntualizaciones teóricas se irán introduciendo durante la visualización y análisis de los vídeos. Una vez vistos los vídeos por grupos de trabajo, cada uno de los subgrupos de tres estudiantes dedicarán el tiempo en clase a la realización de una actividad de contraste propuesta. Una vez terminada la actividad se desarrollará un ejercicio totalmente práctico, en la que los estudiantes deberán aplicar el contenido aprendido durante la clase. Finalmente, los módulos terminarán con un tiempo para exposición y debate de resultados con conclusiones generales. El modelo metodológico definido ha sido un modelo genérico que se aplicará de forma similar con nuevas variables en cada una de las tres sesiones.

Ciclos de Mejora en el Aula (2020). Experiencias de Innovación Docente de la US Esta obra se distribuye con la licencia Creative Commons 


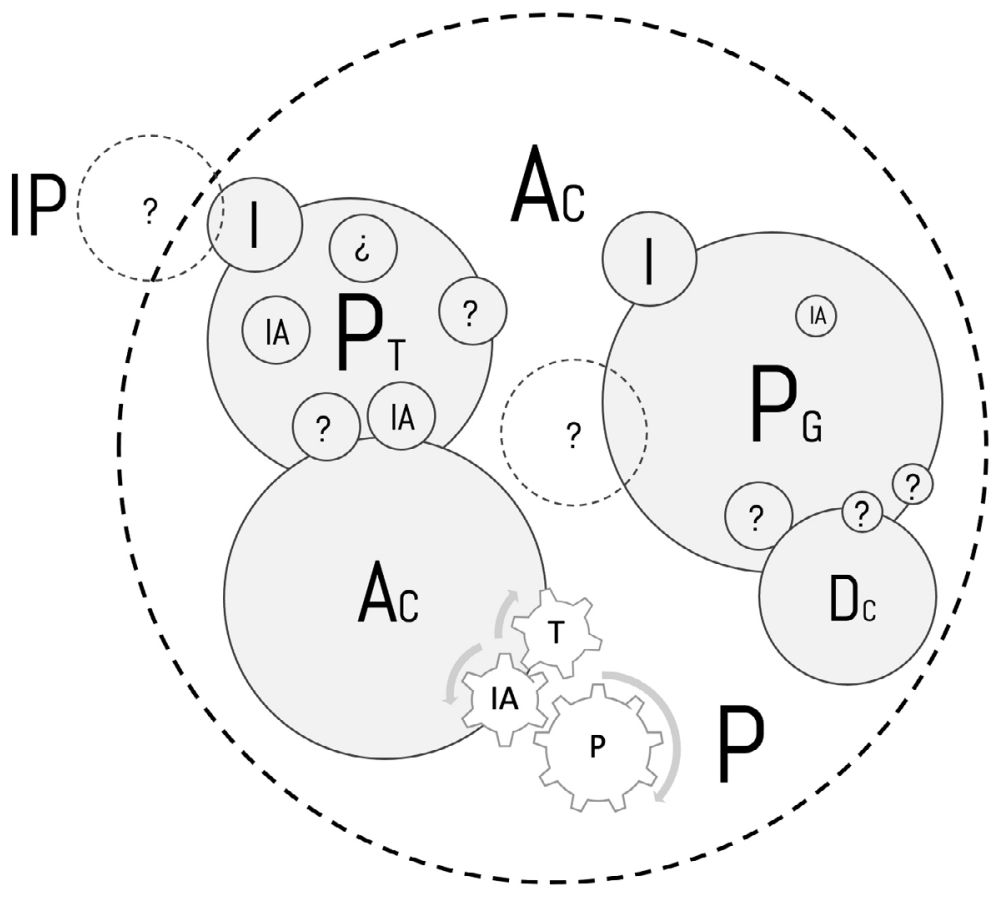

IP: Ideas previas; I: Introducción; Ac: Actividad de Contrase; Pg: Práctica general; Pt: Práctica Teórica; ?: Preguntas; IA: Ideas de los alumnos; Dc: Debate y conclusiones.

Figura 2. Diseño del modelo metodológico.

El Ciclo de Mejora a desarrollar es en tres clases de dos horas. El modelo metodológico por tanto se desarrolla en cada una de las sesiones con la secuencia de actividades diseñadas que se detallan y temporalizan a continuación.

Ciclos de Mejora en el Aula (2020). Experiencias de Innovación Docente de la US Esta obra se distribuye con la licencia Creative Commons 
Tabla 2. Ejemplo genérico de secuencia detallada y temporalizada de las actividades diseñadas.

\begin{tabular}{|c|c|c|c|}
\hline Sesión & Actividad & Descripción & Tiempo \\
\hline 1 & Introducción & $\begin{array}{l}\text { Primeros minutos para preguntar } \\
\text { dudas, cuando se considera } \\
\text { interesante se resuelven las } \\
\text { dudas para el resto de la clase, } \\
\text { ver materiales. Comienza la clase } \\
\text { con la explicación del modelo de } \\
\text { aprendizaje que se va a aplicar } \\
\text { en la sesión. Planteamiento de } \\
\text { problema inicial. A continuación, } \\
\text { se explica la primera actividad } \\
\text { inicial a realizar. }\end{array}$ & $10 \min$ \\
\hline 2 & Práctica Teórica & $\begin{array}{l}\text { Visualización de vídeos realizados } \\
\text { previamente como material } \\
\text { complementario. Se trata de vídeos } \\
\text { resumen del contenido teórico } \\
\text { concisos y atractivos. Los vídeos } \\
\text { recogen el contenido teórico } \\
\text { principal y esencial reduciendo } \\
\text { el tiempo de } 90 \text { a } 20 \text { minutos } \\
\text { aproximadamente. }\end{array}$ & $20 \mathrm{~min}$ \\
\hline 3 & $\begin{array}{l}\text { Actividad de } \\
\text { Contraste }\end{array}$ & $\begin{array}{l}\text { Tras el análisis de los vídeos, } \\
\text { cada alumno contesta un breve } \\
\text { formulario web individualmente; } \\
\text { cada subgrupo de tres alumnos } \\
\text { deberá realizar un mapa de } \\
\text { contenidos de un concepto } \\
\text { determinado y su aplicación en } \\
\text { la arquitectura en las sesiones } \\
1 \text { y 3. El trabajo se realizará con } \\
\text { libertad total de recursos, gráficos, } \\
\text { textuales o de materialidad. En } \\
\text { la sesión } 2 \text { realizarán en grupo el } \\
\text { diseño de tres tipos de sistemas } \\
\text { constructivos propuestos. }\end{array}$ & $30 \mathrm{~min}$ \\
\hline 4 & $\begin{array}{l}\text { Explicación } \\
\text { Práctica }\end{array}$ & $\begin{array}{l}\text { Se procede a la explicación de la } \\
\text { práctica genérica de la sesión por } \\
\text { parte del profesor. }\end{array}$ & $5 \mathrm{~min}$ \\
\hline
\end{tabular}

Ciclos de Mejora en el Aula (2020). Experiencias de Innovación Docente de la US Esta obra se distribuye con la licencia Creative Commons Reconocimiento-NoComercial-SinObraDerivada Internacional (CC BY-NC-ND 4.0.) 


\begin{tabular}{|c|c|c|c|}
\hline 5 & $\begin{array}{l}\text { Práctica } \\
\text { General }\end{array}$ & $\begin{array}{l}\text { Sesión 1: cada grupo elabora una } \\
\text { probeta de } 4 \times 4 \times 16 \mathrm{~cm} \text { de pasta de } \\
\text { yeso, otra de mortero de cal y otra } \\
\text { de mortero de cemento (Figura 3a). } \\
\text { Sesión 2: montaje de diferentes } \\
\text { sistemas constructivos in situ. } \\
\text { Forjado, cerramiento y cubierta } \\
\text { previamente diseñados en } \\
\text { actividad de contraste (Figura 3b). } \\
\text { Sesión 3: Competición en el ensayo } \\
\text { de probetas realizadas en la primera } \\
\text { sesión. Se romperán las probetas a } \\
\text { flexión y compresión en la prensa } \\
\text { hidráulica con clasificación por } \\
\text { grupos con premio en evaluación } \\
\text { de prácticas. }\end{array}$ & $30 \mathrm{~min}$ \\
\hline 6 & Debate & $\begin{array}{l}\text { Tras finalizar la práctica, los } \\
\text { últimos minutos se dedican a la } \\
\text { exposición rápida de algún trabajo } \\
\text { seleccionado, fomentando el } \\
\text { debate entre alumnos. Finalmente, } \\
\text { el profesor recalca las conclusiones } \\
\text { principales del contenido tratado } \\
\text { en la sesión. }\end{array}$ & $25 \mathrm{~min}$ \\
\hline
\end{tabular}

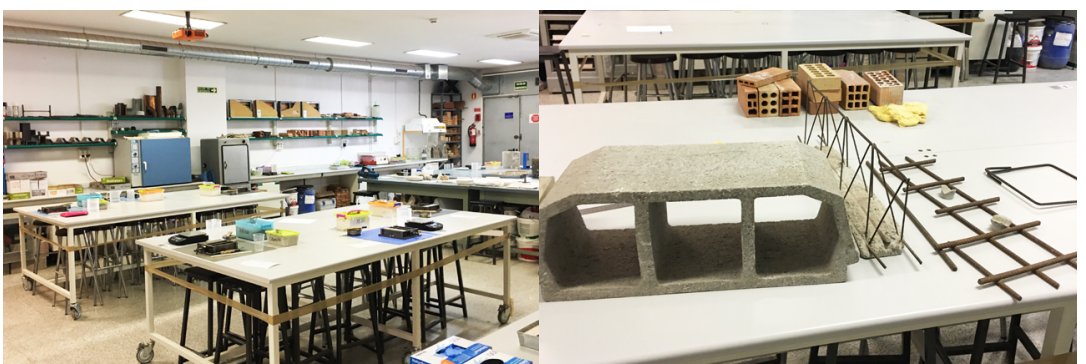

(a)

(b)

Figura 3. Materiales y puestos para la elaboración de la práctica.

Ciclos de Mejora en el Aula (2020). Experiencias de Innovación Docente de la US Esta obra se distribuye con la licencia Creative Commons Reconocimiento-NoComercial-SinObraDerivada Internacional (CC BY-NC-ND 4.0.) 
Las preguntas del cuestionario inicial y final se han diseñado de tal forma que las respuestas nos permitan conocer el estado de conocimiento de los estudiantes, que no se respondan de forma extensa o al azar, de esta forma se podrán detectar sus ideas previas reales sobre la materia. Las cuestiones además se relacionan directamente con los contenidos abordados durante el proceso de aprendizaje. El cuestionario inicial se realiza en la clase previa a la aplicación del CIMA, donde se aprovecha para ponerles en situación de modelo de enseñanza que se va a llevar a cabo en las prácticas de laboratorio. El cuestionario lo realizan los 27 alumnos presentes en el aula, prácticamente la totalidad de los alumnos matriculados mediante formulario web (Figura 4). El cuestionario final se realiza tras la aplicación completa del Ciclo de Mejora.

Ciclos de Mejora en el Aula (2020). Experiencias de Innovación Docente de la US Esta obra se distribuye con la licencia Creative Commons 


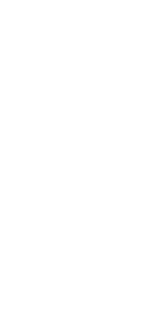

\section{Prácticas Laboratorio Construcción 1}

Cuestionario Inicial Prácticas de Laboratorio Construcción 1

*Obligatorio

Dirección de correo electrónico *

Tu dirección de correo electrónico

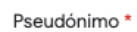

Tu respuesta

¿Qúe entiendes por "TECTÓNICA"? *

Tu respuesta

¿Dónde puedes encontrar yeso, cal y cemento en un edificio? *

Tu respuesta

¿Sabes la diferencia entre pasta, mortero y hormigón? *

Tu respuesta

¿Qué entiendes por forjado? Indica elementos constructivos utilizados para su construcción? *

Tu respuesta

¿Qué es la envolvente del edificio? Indica capas y función de ellas. *

Tu respuesta

Figura 4. Cuestionario Inicial

Ciclos de Mejora en el Aula (2020). Experiencias de Innovación Docente de la US Esta obra se distribuye con la licencia Creative Commons 


\section{Aplicación del CIMA}

\section{Diario de las sesiones}

Previo al desarrollo del CIMA, se les ha enviado un correo con la información y el contenido adicional preparado para la clase. Se trata de los vídeos descritos en el diseño del modelo metodológico. En cada una de las tres sesiones deberán analizar tres vídeos cortos de 5 minutos aproximadamente con el resumen teórico, así como la realización de un breve cuestionario web sobre el contenido del mismo. A continuación, se procede a relatar de forma resumida la experiencia en cada una de las sesiones.

La primera sesión comienza de forma diferente a lo habitual en otros cursos debido al funcionamiento del laboratorio en tiempos de COVID. En este caso los alumnos esperan en su aula de cada día y el profesor de laboratorio acude al aula. Durante los primeros minutos se les resuelven las dudas sobre el contenido del correo enviado previamente, se les explica el funcionamiento del laboratorio y desarrollo de la clase. Se les plantea de forma conjunta el contenido genérico de la práctica y se les explica cada una de las actividades que van a desarrollar. Debido a la imposibilidad de aforo del laboratorio, los últimos días me veo obligado a replantear los tiempos y actividades diseñadas. Los alumnos permanecerán en el aula y vendrán al laboratorio en tres grupos de nueve integrantes, mientras el resto permanece en el aula controlados por su profesor de teoría. Es por esto que la práctica general y teórica se realizan simultáneamente por los diferentes grupos en diferente orden. Mientras el primer grupo me acompaña al laboratorio el resto permanece realizando las actividades propuestas en el aula, de esta forma permanezco durante 1:30 h en el laboratorio, 30 minutos por cada grupo. Paralelamente, los alumnos tienen una hora de tiempo total para trabajar en clase en la visualización de vídeos (20

Ciclos de Mejora en el Aula (2020). Experiencias de Innovación Docente de la US Esta obra se distribuye con la licencia Creative Commons 
min), desarrollo de la actividad de contraste (30 min) y la realización de un cuestionario web (10 min) que se diseñó los días previos para poder completar el tiempo que yo permanecía con el resto de compañeros en el laboratorio.

Con respecto a la actividad, al tener más grupos hice una prueba los días previos para ensayar tiempos y pude comprobar que no visualizaban los vídeos con la atención que esto requería, por lo que el cuestionario les podía obligar a analizar el contenido de forma más exhaustiva.

Durante la realización de la práctica (mientras están elaborando las probetas) aproveché para ir haciendo comentarios y preguntas generales sobre los contenidos. Fue muy satisfactorio ver como en el primer subgrupo que hizo la práctica (no habían visto los vídeos ni cuestionario todavía) apenas sabían de lo que hablaba, no contestaban preguntas y tuve que hacer un monólogo, mientras que con el segundo subgrupo (había visto los vídeos, pero no habían hecho el cuestionario) ya tenían ideas sueltas del contenido de la práctica. Por último, cuando el tercer subgrupo vino al laboratorio, prácticamente me dediqué a preguntar aspectos relacionados con el contenido de la práctica, entre unos y otros lo sabían responder prácticamente todo. ¡Los vídeos y cuestionario habían servido! Los últimos minutos de la clase vuelvo al aula donde se seleccionó uno de los mapas conceptuales más avanzados para comentarlo en clase. Para mi sorpresa la participación fue mejor de la esperada. Se comentaron dudas surgidas del contenido y se debatió sobre algunos contenidos concretos.

Los aspectos generales del funcionamiento se repiten en las tres sesiones, y el sentimiento de qué es lo que realmente están aprendiendo también. La segunda sesión fue un proceso similar al anterior, pero con contenidos diferentes, comenzamos con aspectos generales y resolviendo dudas de la práctica y trabajos anteriores y se les recuerda cómo va a funcionar la clase. En este caso la práctica a realizar en el laboratorio se tiene que reducir en cuanto al contenido, ya que sólo podía estar media hora con cada

Ciclos de Mejora en el Aula (2020). Experiencias de Innovación Docente de la US Esta obra se distribuye con la licencia Creative Commons 
grupo y tenía planteados 45 minutos, por esto, el debate final aproveché para hacerlo brevemente con cada grupo en el propio laboratorio. Por otra parte, la actividad cambia al no tener en el laboratorio los materiales necesarios para todos los alumnos. No se permitía que cada alumno utilizara los materiales que requiere para montar los diferentes sistemas constructivos propuestos ya que no había tiempo ni personal para desinfectar cada producto utilizado. Conseguí solventar esto colocando a los alumnos alrededor de una gran mesa de trabajo y yo fui en este caso el que fue construyendo los diferentes sistemas. Siempre procuré que fueran ellos los que me indicaran que producto o material coger y donde colocarlo. Este proceso les resultó bastante divertido, la participación fue muy buena. De nuevo el tercer grupo que visitó el laboratorio tenía un mayor conocimiento de los productos y de los diferentes sistemas constructivos.

El funcionamiento de la tercera clase fue de nuevo similar a las anteriores, esto se debe a que el modelo metodológico se ha diseñado de forma cíclica para cada uno de los tres contenidos estructurantes principales (uno por cada sesión). En este caso, el número de probetas realizadas era mayor que otros años debido a que se han realizado de forma individual en el laboratorio y no dio tiempo a completar la competición, no obstante, al resultarles esto muy divertido les hemos prometido romper el resto y comunicarles los resultados finales. Al comprobar que no iba a dar tiempo se decidió ensayar al menos alguna probeta de cada uno de los tres conglomerantes estudiados, para así poder comentar aspectos teóricos genéricos en función de los resultados. En este caso la práctica que desarrollamos en el laboratorio no estaba relacionada con el contenido teórico de los vídeos y las actividades diseñadas por lo que se aprovechó el debate final para exponer algunos de los mapas de contenido y recalcar aspectos generales que consideraba importantes para ellos.

Ciclos de Mejora en el Aula (2020). Experiencias de Innovación Docente de la US Esta obra se distribuye con la licencia Creative Commons 
La sensación general de la clase ha sido muy satisfactoria, tanto para mí que me ha resultado mucho más fácil organizar la clase, como para los alumnos, a los cuales he visto mucho más entretenidos y con conocimientos que en mi primera experiencia el curso anterior. Destacaría sobre todo el nivel de participación y motivación en relación al curso anterior. El trabajo en el aula funcionó muy bien, al menos en los resultados eso se mostraba. El CIMA ha permitido reducir el contenido teórico y hacerlo más atractivo para los alumnos. El objetivo de convertir la clase práctica en una práctica real creo que se ha cumplido sobradamente; en ningún momento ha habido alumnos en sillas escuchando un discurso contado por el profesor.

Las mayores dificultades durante la aplicación del segundo CIMA fueron previas a la aplicación del mismo. Tener que adaptar algunas actividades al funcionamiento del laboratorio durante el COVID me supuso alguna preocupación previa que fue solventada en la medida de lo posible. Lo más preocupante era durante la primera sesión no poder controlar al grupo en general al estar partido en tres, pero el funcionamiento fue muy bueno. El mayor agobio ha sido controlar el tiempo de las actividades para que todo fuera como estaba planteado.

\section{Evaluación del aprendizaje de los estudiantes}

Para realizar el análisis comparativo y evaluar el aprendizaje de los estudiantes se ha realizado el mismo cuestionario al inicio y al final del CIMA. A continuación, se muestran las escaleras de aprendizaje para una de las preguntas realizadas en el cuestionario, así como el cuadro de evolución por estudiante en cada pregunta. Para esto se han numerado los sujetos participantes del 1 al 27; esto ha sido posible por su identificación previa mediante un mismo pseudónimo en ambos cuestionarios.

Ciclos de Mejora en el Aula (2020). Experiencias de Innovación Docente de la US Esta obra se distribuye con la licencia Creative Commons 


\section{¿Qué entiendes por forjado? Indica elementos cons- tructivos utilizados para su construcción}

\begin{tabular}{|c|c|c|}
\hline $\begin{array}{c}\text { Modelos de ideas } \\
\text { detectados }\end{array}$ & $\begin{array}{c}\text { Numeración Sujetos } \\
\text { Cuestionario inicial }\end{array}$ & $\begin{array}{c}\text { Numeración Sujetos } \\
\text { Cuestionario final }\end{array}$ \\
\hline No sabe / No contesta & $1,3,6,12,13,24$ & $3,6,13$ \\
\hline $\begin{array}{c}\text { Relaciona el concepto } \\
\text { con la arquitectura }\end{array}$ & $\begin{array}{c}4,1014,15,17,18,19,22, \\
23,25,26,27\end{array}$ & $1,4,24$ \\
\hline $\begin{array}{c}\text { Relaciona el concepto } \\
\text { con elemento } \\
\text { estructural }\end{array}$ & $7,9,11,16$ & $\begin{array}{c}10,12,14,15,17,18,19, \\
22,23,25,26\end{array}$ \\
\hline $\begin{array}{c}\text { Define el concepto e } \\
\text { indica productos que lo } \\
\text { forman }\end{array}$ & $2,5,8,20,21$ & $2,5,7,8,9,11,16,20$, \\
21,27
\end{tabular}

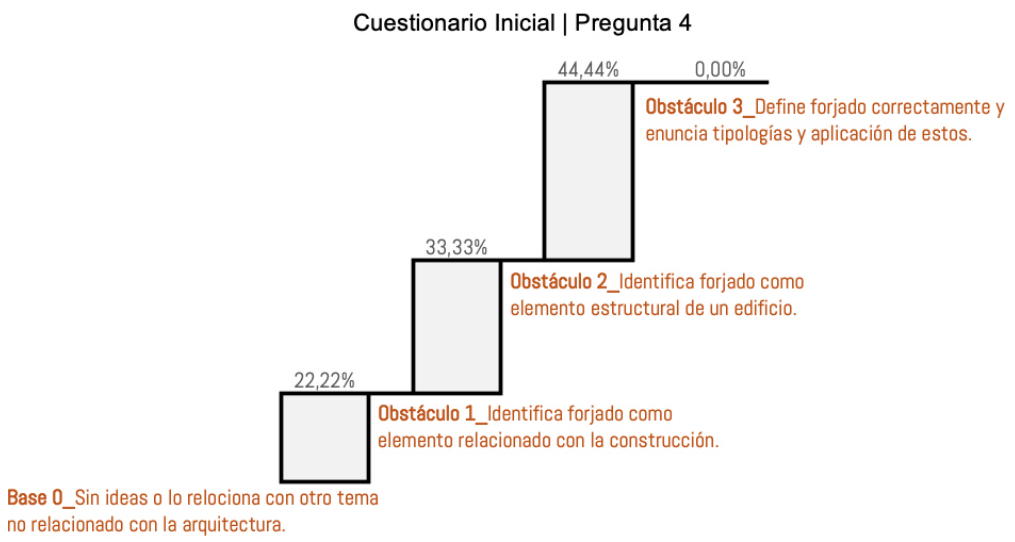

Cuestionario Final | Pregunta 4

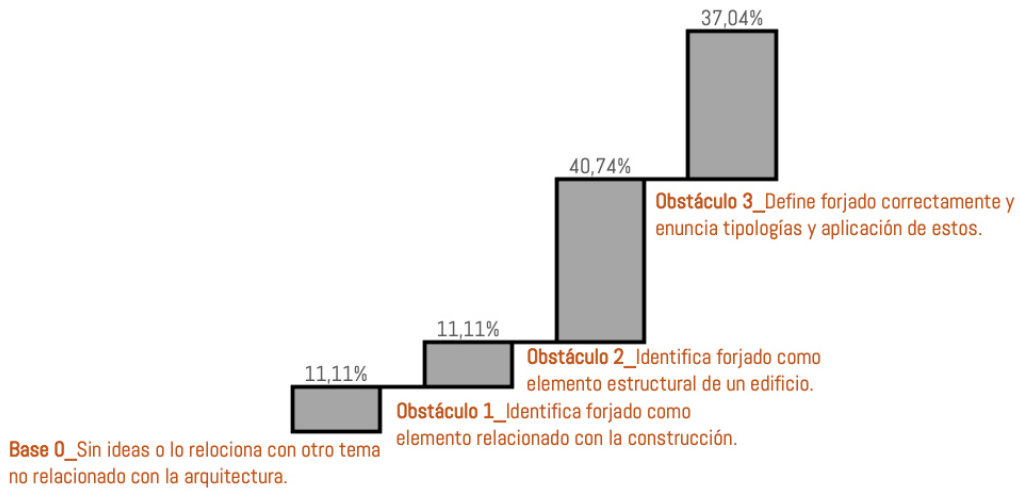

Figura 5. Análisis comparativo de aprendizaje inicial y final de los estudiantes.

Ciclos de Mejora en el Aula (2020). Experiencias de Innovación Docente de la US CC $\Theta$ Esta obra se distribuye con la licencia Creative Commons 
Las escaleras parten de una base en el que el alumno apenas tiene ideas relacionadas con el contenido abordado en la pregunta correspondiente. Posteriormente se plantean una serie de obstáculos que deberán salvar durante el proceso de aprendizaje, con un desarrollo cada vez más complejo. En el primer obstáculo el alumno consigue identificar el contenido como un elemento relacionado con la construcción, sin alcanzar unos mínimos de exigencia. El segundo obstáculo será identificar el forjado como un elemento estructural del edificio, siendo el último el que permita alcanzar la capacidad de definir correctamente el contenido cuestionado, así como identificar diferentes tipologías y casos de aplicación. Mientras que en el cuestionario inicial la mayoría de la clase se encontraba en los dos primeros niveles, el cuestionario final indica que prácticamente el $80 \%$ de la clase ha alcanzado los mínimos de exigencia planteados, destacando un 37\% de alumnos con el concepto totalmente claro.

\section{Evaluación del CIMA}

Analizando el aprendizaje de los alumnos durante la aplicación del Ciclo de Mejora ha permitido conocer al conjunto de la clase de forma más exhaustiva y poder diseñar las actividades de forma más adecuada. Los resultados adquiridos y comentarios de los propios estudiantes han sido muy satisfactorios, bastante más que en cursos anteriores.

Los contenidos empleados para la enseñanza han sido sintetizados y estructurados gracias al trabajo previo sobre estos. Los procesos previos de análisis y sintesis han permitido centrar el foco del proceso de aprendizaje en lo realmente importante.

El diseño del modelo metodológico a aplicar durante el CIMA, pero, sobre todo, representar el modelo metodológico habitual es sin duda el aspecto más enriquecedor que me llevo. Me ha permitido ser consciente de que lo 
que se impartía como una práctica de laboratorio no era un proceso realmente práctico, simplemente era una clase teórica impartida en otra sala. Diseñar posteriormente una metodología que permita modificar esto ha sido fundamental. Su relación con el mapa de contenidos a través de diferentes preguntas claves y la secuencia de actividades ha enfatizado la importancia de la metodología diseñada.

El diagnóstico inicial fue fundamental para el diseño de las actividades y conocer a qué vamos a enfrentarnos, sin embargo, creo que con algo más de tiempo me hubiera permitido diseñar alguna actividad de forma diferente, recalcando algún concepto a priori concebido como algo más secundario. La identificación de los obstáculos ha sido fundamental para conocer hasta donde podian llegar y como avanza la clase. El análisis comparativo de las escaleras de aprendizaje ha sido muy motivante al ver el resultado de aprendizaje obtenido.

Ciclos de Mejora en el Aula (2020). Experiencias de Innovación Docente de la US Esta obra se distribuye con la licencia Creative Commons 
Palabras claves: Construcción, arquitectura, docencia universitaria, experimentación docente universitaria.

\section{Referencias bibliográficas}

Bain, K. (2007). Lo que hacen los mejores profesores universitarios. Universidad de Valencia.

Blandón González, B. (2019). Propuesta del nuevo modelo didáctico aplicado en construcción. Jornadas De Formación E Innovación Docente Del Profesorado, (1), 5879. https://doi.org/10.12795/jdu.2018.i01.03

Finkel, D. L. (2008). Dar clase con la boca cerrada. Valencia: Universidad de Valencia.

Navarro Medina, E. y Porlán Ariza, R. (2020). Ciclos de mejora en el aula año 2019. Experiencias de innovación docente de la Universidad de Sevilla. Universidad de Sevilla.

Porlán Ariza, R. (2008). El diario de clase y el análisis de la práctica. Andalucia: Averroes. Red Telemática Educativa de Andalucía.

Porlán Ariza, R. (2017). Enseñanza Universitaria. Cómo mejorarla. Madrid: Morata.

Ciclos de Mejora en el Aula (2020). Experiencias de Innovación Docente de la US Esta obra se distribuye con la licencia Creative Commons 